\title{
Network Reconfiguration For Loss Reduction Of A Radial Distribution System
}

\author{
Laxmi. M. Kottal ${ }^{1}$, Radhika priyadarshini ${ }^{2}$, Dr R Prakash ${ }^{3}$ \\ M.Tech Sholar, Department of EEE, Acharya Institute of Technology,Bengaluru, India ${ }^{1}$ \\ Research scholar, Department of EEE, Acharya Institute of Technology,Bengaluru, India Country ${ }^{2}$ \\ Professor, Department of EEE, Acharya Institute of Technology,Bengaluru, India ${ }^{3}$
}

\begin{abstract}
By using network reconfiguration process many distribution system problems can be solved such as planning, energy restoration and loss minimization. This paper describes about loss reduction in a radial distribution system by doing network reconfiguration using new algorithm i.e. Gravitational Search Algorithm [GSA].GSA considers all the objectives in order to meet the reliability requirements. And because of this the processing time can be reduced also the quality of solution can be improved. The proposed approach has been applied to distribution test systems including IEEE 33 system.
\end{abstract}

Keywords: Network reconfiguration; distribution system; loss minimisation; gravitational search algorithm.

\section{INTRODUCTION}

Distribution systems are designed to operate in a radial configuration only even though it is designed in meshed structure also. Because radial structure has many advantages over meshed structure like simple in construction, operation is simple and initial cost is less.

In a distribution system network reconfiguration is processes that chances the topological structure of feeder by managing the open/close status of the sectionalizing and tie switches in distribution system in order to minimize the losses. Usually sectionalizing switches will be in closed position and that of tie switches will be in open position. Obviously more the number of switches, there will be more options to do reconfiguration of the network in order to get better effects towards minimizing the losses.

Consumer demands vary with time of day, day of the week and season; therefore feeder reconfiguration enables load transfers from heavily to weakly loaded regions. Network reconfiguration can also be used in planning studies, in order to determine the optimal configuration of the network during the overall planning procedure. Furthermore, online configuration management becomes an important part of distribution automation when remote controlled switches are employed [1]. Since a typical distribution system may have hundreds of switches, a combinatorial analysis of all possible options is not a practical proposition. The radiallity constraint and the discrete nature of the switch values prevent the use of classical optimization techniques to solve the reconfiguration problem.

Network reconfiguration is done mainly due to: i.It can provide service to as many as consumer as loops.

possible even in the planned outage condition for maintenance purpose.

ii.Avoid system over loading of network elements by

balancing the loads.

iii.System losses can be reduced.

Ganesh et al. [2] presented paper based on an efficient approach to feeder reconfiguration for loss reduction and voltage profile improvement in unbalanced radial distribution system. Enoque et el. [3] presented paper, how the application of loss minimization in network reconfiguration In distribution system can help to evaluate online reconfiguration benefits in each node of the system. Dan Jiang et al. [4] presented paper on single comprehensive algorithm for distribution system switch reconfiguration and capacitor control. In this paper Simulated Annealing is employed to optimize the switch reconfiguration of distribution system. Zeng Rong et al. [5] used Minimal Nodal Voltage method and Genetic Algorithm for loss minimization in radial distribution system. Chang-Fu change [6] presented a paper to study distribution system operation by Ant Colony Search algorithm (ACSA). The ACSA is relatively new and power full Swarm intelligence method for solving optimization problems. They observed that the results of ACSA are better than SA and GA, and this method is suitable for large scale distribution system.

Yong-Jae Jeon et al. [7] presented paper on loss minimization by Simulated Annealing algorithm which is suitable particularly for large combinatorial optimization problem. A branch exchange type heuristic algorithm has been suggested by Civanlar et al [8], where a simple formula has been derived to determine how a branch exchange affects the losses. In Shirmohammadi and Hong [9] the solution method starts with a meshed distribution system obtained by considering all switches closed. Then the switches are opened successively to eliminate the

\section{PROBLEM FORMULATION}

\section{A. Objective function}

The main objective is to minimize the losses in radial distribution system in order to improve the voltage profile. Min $\mathrm{f}=\min \left(P_{T, \text { loss }}\right)$ 
where $P_{T, \text { loss }}$ is the total real power loss of the system.

\section{B. Constraints}

i. Bus voltage limit.

$$
V_{\min } \leq V_{i} \leq V_{\max }
$$

Where $V_{i}$ is voltage magnitude at the $i^{\text {th }}$ node and $V_{\min }$ and $V_{\max }$ are the allowable voltage limits at the same bus.

$$
\begin{aligned}
& \text { ii. } \quad \text { Feeder limit. } \\
& \left|I_{f, i}\right| \leq I_{f, i}^{\max } \quad \mathrm{i}=1,2 \ldots N_{\text {feeder }}
\end{aligned}
$$

Where $I_{f, i}$ is the current amplitude and $I_{f, i}^{\max }$ is maximum allowable current value in the $i^{\text {th }}$ feeder. And $N_{\text {feeder }}$ is total number of feeders.

iii. Radial structure of network.

$$
N_{\text {branch }}=N_{\text {bus }}-N_{\text {source }}
$$

Where $N_{\text {bus }}$ is the number of buses, $N_{\text {source }}$ is number of substations.

\section{GRAVITATIONAL SEARCH ALGORITHM}

\section{A. Over view of GSA}

GSA was introduced by Rashedi et al. in 2009 and is intended to solve optimization problems. The population based heuristic algorithm is based on the law of gravity and mass interactions. The algorithm is comprised of collection of searcher agents that interact with each other through the gravity force [1]. The agents are considered as objects and their performance is measured by their masses. The gravity force causes a global movement where all objects move towards other objects with heavier masses. The slow movement of heavier masses guarantees the exploitation step of the algorithm and corresponds to good solutions. The masses are actually obeying the law of gravity as shown in Equation (1) and the law of motion in Equation (2).

$$
F=G\left(\frac{M_{1} M_{2}}{R^{2}}\right)
$$

$$
\mathrm{a}=\mathrm{F} / \mathrm{M}
$$

Based on Equation (1), F represents the magnitude of the gravitational force, $\mathrm{G}$ is gravitational constant, $\mathrm{M} 1$ and $\mathrm{M} 2$ are the mass of the first and second objects and $\mathrm{R}$ is the distance between the two objects. Equation (1) shows that in the Newton law of gravity, the gravitational force between two objects is directly proportional to the product of their masses and inversely proportional to the square of the distance between the objects. While for Equation (2), Newton's second law shows that when a force, F, is applied to an object, its acceleration, a, depends on the force and its mass, $M$.

In GSA, the agent has four parameters which are position, inertial mass, active gravitational mass, and passive gravitational mass [1]. The position of the mass represents the solution of the problem, where the gravitational and inertial masses are determined using a fitness function. The algorithm is navigated by adjusting the gravitational and inertia masses, whereas each mass presents a solution. Masses are attracted by the heaviest mass. Hence, the heaviest mass presents an optimum solution in the search space. The steps of GSA are as follows:

Step 1: Agents initialization:

The positions of the $\mathrm{N}$ number of agents are initialized randomly

$x_{i}=\left(x_{i}^{1}, x_{i}^{2}, \ldots x_{i}^{d} \ldots \ldots, x_{i}^{N}\right) \quad$ where $\mathrm{i}=1,2 \ldots \mathrm{N}$

$x_{i}^{d}$ represents the positions of the $i^{\text {th }}$ agent in the $d^{\text {th }}$ dimension, while $\mathrm{n}$ is the space dimension.

Step 2: Fitness evolution and best fitness computation:

For minimization or maximization problems, the fitness evolution is performed by evaluating the best and worst fitness for all agents at each iteration.

Minimization problems:

$$
\begin{gathered}
\text { Best }(\mathrm{t})=\min \text { fit } \mathrm{j}(\mathrm{t}) \\
\mathrm{J} \in\{1 \ldots . N\} \\
\text { Worst }(\mathrm{t})=\max \text { fit } \mathrm{j}(\mathrm{t}) \\
\mathrm{J} \in\{1 \ldots . N\}
\end{gathered}
$$

Maximization problems:

$$
\begin{gathered}
\operatorname{Best}(\mathrm{t})=\max \text { fit } \mathrm{j}(\mathrm{t}) \\
\mathrm{J} \in\{1 \ldots . N\} \\
\text { Worst }(\mathrm{t})=\min \text { fit } \mathrm{j}(\mathrm{t}) \\
\mathrm{J} \in\{1 \ldots . N\}
\end{gathered}
$$

fit $\mathrm{j}(\mathrm{t})$ represents the fitness value of the $j^{\text {th }}$ agent at iteration $t$, best $(t)$ and $\operatorname{worst}(t)$ represents the best and worst fitness at iteration $t$.

Step 3: Gravitational constant $(\mathrm{G})$ computation:

Gravitational constant $\mathrm{G}$ is computed at iteration $\mathrm{t}$

$$
G(t)=G_{0} e^{(-\propto t / T)}
$$

$G_{0}$ and $\propto$ are initialized at the beginning and will be reduced with time to control the search accuracy. $\mathrm{T}$ is the total number of iterations.

Step 4: Masses of the agents' calculation:

Gravitational and inertia masses for each agent are calculated at iteration $\mathrm{t}$. 


$$
\begin{aligned}
m_{i}(t) & =\frac{\text { fit }_{i}(t)-\operatorname{worst}(t)}{\operatorname{best}(t)-\operatorname{worst}(t)} \\
M_{i}(t) & =\frac{m_{i}(t)}{\sum_{j=1}^{N} m_{j}(t)}
\end{aligned}
$$

Step 5: Accelerations of agents' calculation:

Acceleration of the $i^{\text {th }}$ agents at iteration $\mathrm{t}$ is computed.

$$
a_{i}^{d}(t)=\frac{F_{i}(t)}{M_{i}(t)}
$$

$F_{i}^{d}(t)$ is the total force acting on $i^{t h}$ agent calculated as:

$$
F_{i}^{d}(t)=\sum_{i \epsilon K_{\text {best }}} \operatorname{rand}_{j} F_{i j}^{d}(t)
$$

Kbest is the set of first $\mathrm{K}$ agents with the best fitness value and biggest mass. Kbest will decrease linearly with time and at the end there will be only one agent applying force to the others.

$F_{i j}^{d}(t)$ is computed as the following equation:

$F_{i j}^{d}(t)=\mathrm{G}(\mathrm{t}) \frac{M_{i}(t) M_{j}(t)}{R_{i j}(t)+\varepsilon}\left(\left(x_{j}^{d}(t)-x_{i}^{d}(t)\right)\right.$

$F_{i j}^{d}(t)$ is the force acting on agent $\mathrm{i}$ from agent $\mathrm{j}$ at $d^{\text {th }}$ dimension and $i^{\text {th }}$ iteration. $R_{i j}(t)$ is the Euclidian distance between two agents $i$ and $j$ at iteration t. $G(t)$ is the computed gravitational constant at the same iteration while $\varepsilon$ is a small constant.

Step 6: Velocity and positions of agents:

Velocity and the position of the agents at next iteration $(t+1)$ are computed based on the following equations:

$$
\begin{gathered}
v_{i}^{d}(t+1)=\operatorname{rand}_{i} v_{i}^{d}(t)+a_{i}^{d}(t) \\
x_{i}^{d}(t+1)=x_{i}^{d}(t)+v_{i}^{d}(t+1)
\end{gathered}
$$

Step 7: Repeat steps 2 to 6

Steps 2 to 6 are repeated until the iterations reach their maximum limit. The best fitness value at the final iteration is computed as the global fitness while the position of the corresponding agent at specified dimensions is computed as the global solution of that particular problem. Fig. 1 shows the flowchart of GSA.

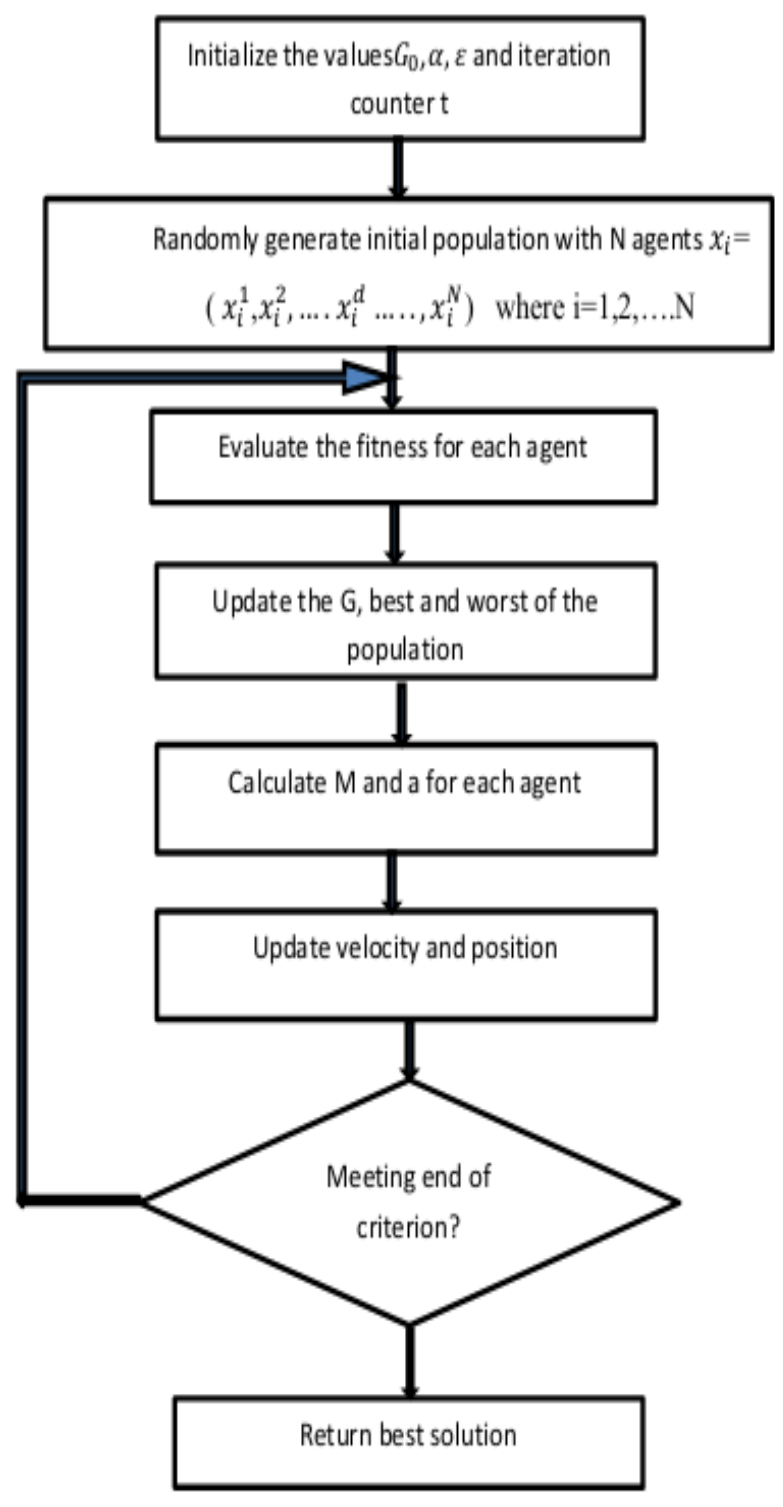

Fig. 1 flowchart of GSA

\section{RESULT AND ANALYSIS}

For 33bus-test system before doing network reconfiguration losses in 33 bus test system was $79 \mathrm{KW}$ and after doing reconfiguration losses becomes $60 \mathrm{KW}$. So after doing network reconfiguration the losses were reduced up to $23.6 \%$ for half load condition.

\begin{tabular}{|l|l|l|l|}
\hline & $\begin{array}{l}\text { Total } \\
\text { loss(KW) }\end{array}$ & $\begin{array}{l}\text { Loss after } \\
\text { reconf (KW) }\end{array}$ & $\begin{array}{l}\text { \%loss } \\
\text { reduction(KW) }\end{array}$ \\
\hline $\begin{array}{l}\text { Case 1: } \\
\text { half load }\end{array}$ & 78.74 & 60.1625 & $23.6 \%$ \\
\hline $\begin{array}{l}\text { Case 2: } \\
\text { full load }\end{array}$ & 360.4 & 264.46 & $26.6 \%$ \\
\hline $\begin{array}{l}\text { Case 3: } \\
\text { over load }\end{array}$ & 970.8 & 661.914 & $31.817 \%$ \\
\hline
\end{tabular}




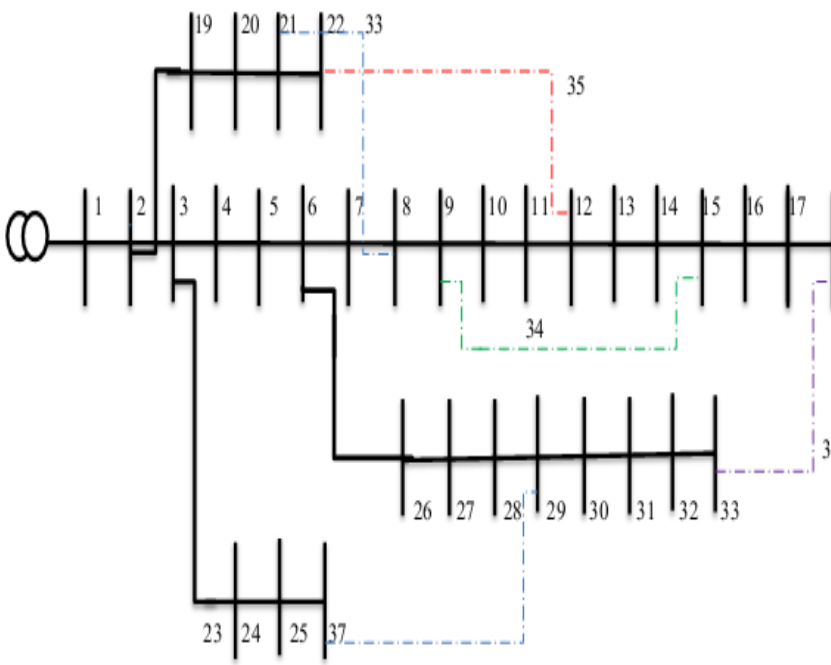

Fig 2 single line diagram of 33 bus test system

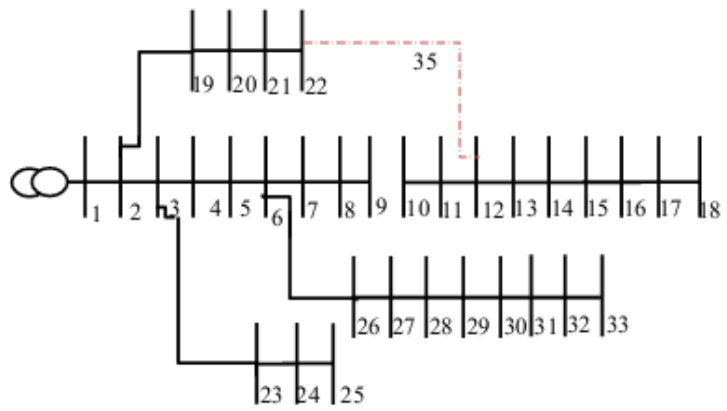

Fig 3 single line diagram of 33 bus test system after reconfiguration

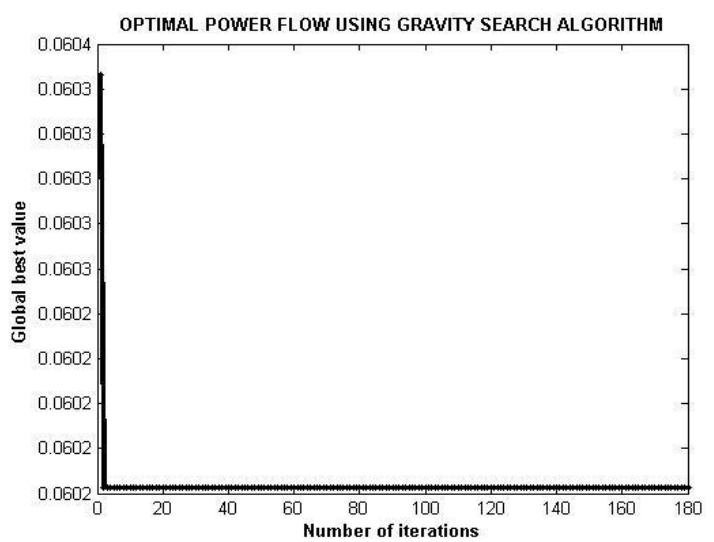

Case 1

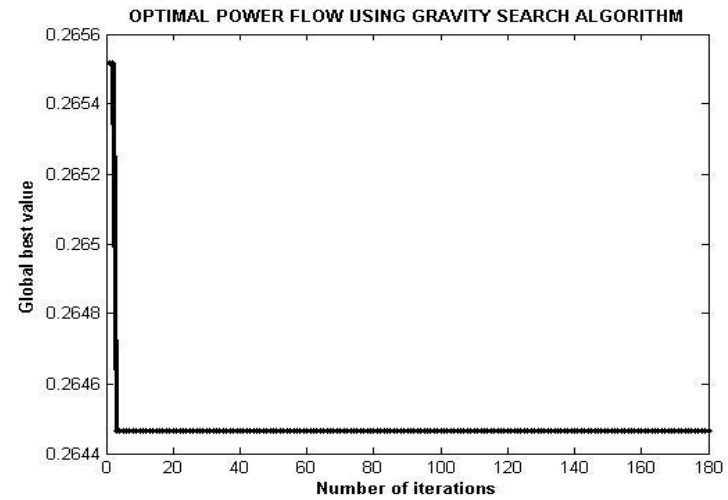

Case 2

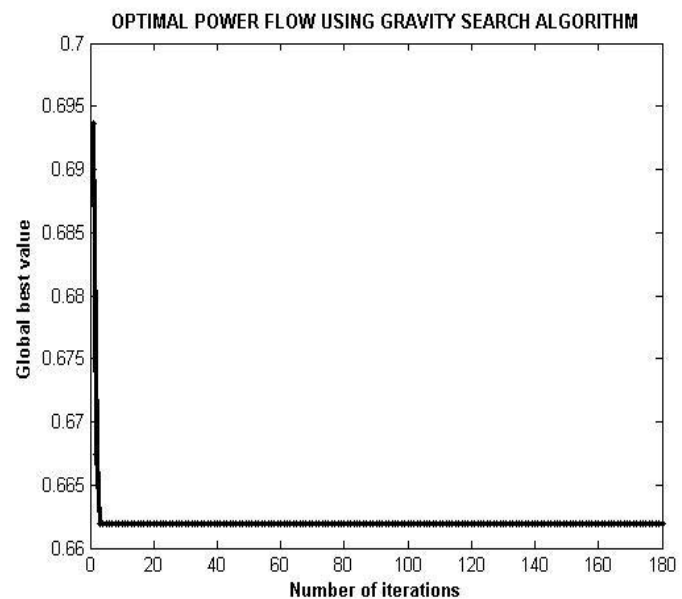

Case 3

\section{CONCLUSION}

A new powerful evolutionary algorithm has been presented in this paper for the DFR. The proposed DFR problem consists of minimizing the power loss. The considered constraints including the bus voltage limit, feeder limit and radial structure of the network are within their admissible ranges in this approach. The algorithm has been successfully tested for 33 bus test system for different loading condition in distribution network.

Main advantage of using GSA:

- The presented optimization algorithm has low computational time, allowing its application in the context of large scale distribution systems.

- The considered ENS objective function accompanied by other objectives paves the way to have a reliable and economic condition in distributed systems.

\section{REFERENCES}

[1] López E., Opazo, H., García L. and Bastard, P., "Online Reconfiguration considering Variability Demand: Applications to Real Networks", IEEE Transactions on Power Systems, 2004, vol. 19 , no. 1 , pp. 549-553.

[2] G. Eason, B. Noble, and IN. Sneddon, "On certain integrals of Lipschitz-Hankel type involving products of Bessel functions," Phil. Trans. Roy. Soc. London, vol. A247, pp. 529-551, April 1955. (references) 
[3] J. Clerk Maxwell, A Treatise on Electricity and Magnetism, 3rd ed., vol. 2. Oxford: Clarendon, 1892, pp.68-73.

[4] I.S. Jacobs and C.P. Bean, "Fine particles, thin films and exchange anisotropy," in Magnetism, vol. III, G.T. Rado and H. Suhl, Eds. New York: Academic, 1963, pp. 271-350.

[5] K. Elissa, "Title of paper if known," unpublished.

[6] R. Nicole, "Title of paper with only first word capitalized," J. Name Stand. Abbrev., in press

[7] Y. J. Jean and J. C. Kim, "An efficient simulated annealing algorithm for network reconfiguration in large-scale distribution systems," IEEE Trans. Power Del., vol. 17, no. 4, pp. 1070-1078, Oct. 2002.

[8] Civanlar, S., Grainger, J. J., Yin, H. and Lee, S. S. H. "Distribution Feeder Reconfiguration for Loss Reduction", IEEE Transactions on Power Delivery, 1988, vol. 3, no. 3, pp. $1217-1223$.

[9] Shirmohammadi, D. and Hong, H. W., "Reconfiguration of Electric Distribution for Resistive Line Loss Reduction", IEEE Transactions on Power Delivery, 1989, vol. 4, no. 2, pp. $1492-$ 1498

[10] Y. Yorozu, M. Hirano, K. Oka, and Y. Tagawa, "Electron spectroscopy studies on magneto-optical media and plastic substrate interface," IEEE Transl. J. Magn. Japan, vol. 2, pp. 740741, August 1987 [Digests 9th Annual Conf. Magnetics Japan, p. 301, 1982].

[11] M. Young, The Technical Writer's Handbook. Mill Valley, CA: University Science, 1989. 